\title{
Multiple Vitelliform Lesions as a Retinal Manifestation of Alport Syndrome
}

\author{
Kathrine O. Eriksen ${ }^{a} \quad \varnothing y s t e i n$ Kalsnes Jørstad ${ }^{a, b}$ \\ ${ }^{a}$ Department of Ophthalmology, Oslo University Hospital, Oslo, Norway; ${ }^{b}$ Faculty of \\ Medicine, University of Oslo, Oslo, Norway
}

\section{Keywords}

Alport syndrome $\cdot$ Multiple vitelliform lesions · Retina · Type IV collagen · Bruch's membrane

\begin{abstract}
Alport syndrome is associated with various ocular phenotypic features, including several retinal manifestations. The purpose of this case report was to describe a case of multiple vitelliform lesions in Alport syndrome. This particular finding has, to our knowledge, not been reported previously. A 63-year-old man with known Alport syndrome presented with symptomatic, bilateral anterior lenticonus. Fundoscopic examination revealed multiple vitelliform lesions, which were symmetrically distributed at the posterior poles. Additional retinal findings included an irregular foveal contour and central macular thinning in both eyes, as well as a multilayered retinoschisis in the left eye. The underlying pathophysiology of the vitelliform lesions may be a dysfunctional Bruch's membrane.

(C) 2020 The Author(s)

Published by S. Karger AG, Basel
\end{abstract}

\section{Introduction}

Alport syndrome (AS), named after the South African physician Arthur Cecil Alport (1880-1959), is a genetically heterogeneous disorder that results from mutations in various genes encoding type IV collagen $[1,2]$. Collagen IV is the most abundant protein in basement membranes of the kidney, cochlea, and eye. Correspondingly, structural abnormalities at these 
sites lead to the typical phenotypic characteristics of AS in the form of renal disease, sensorineural hearing loss, and ocular anomalies. While the most prevalent ophthalmic features of AS are anterior lenticonus and dot-and-fleck retinopathy, several less common corneal and retinal manifestations have been identified. We here present a case of AS with multiple vitelliform lesions, a finding that, to our knowledge, has not been reported previously in AS.

\section{Case Report}

A 63-year-old Caucasian male with known AS was referred to our retina service for evaluation of fundus abnormalities noted by a general ophthalmologist. The patient's diagnosis had formerly been made on the combination of a family history suggesting X-linked AS, typical clinical signs, and a positive kidney biopsy. As far as we know, no family member had undergone genetic testing.

The patient developed end-stage renal disease at a young age and had undergone two renal transplantations. He also suffered from profound hearing loss. In addition to AS, he had a 10-year history of well-controlled diabetes mellitus type 2, without signs of diabetic retinopathy. His medical treatment comprised immunosuppressants (azathioprine, prednisone, and cyclosporine), an antihypertensive agent (nifedipine), a statin (fluvastatin), benign prostatic hyperplasia medication (dutasteride and tamsulosin), and oral antidiabetics (glipizide and linagliptin).

An ophthalmologist had previously examined the patient at 24 years of age. At that time, best-corrected visual acuity (BCVA) was 6/6 in both eyes, and no findings were noted on general eye examination. Over the past 5 years, the patient had experienced progressive myopia and sought advice of the referring ophthalmologist when poor vision disqualified for renewing his driving license.

On clinical examination, BCVA was $6 / 24$ in the right eye (6/12 with pinhole) and 6/48 in the left eye (6/15 with pinhole). The refractive error was $-13 \mathrm{dpt} /-1.50 \mathrm{dpt} \times 90$ in the right eye and $-10 \mathrm{dpt} /-1.0 \mathrm{dpt} \times 100 \mathrm{in}$ the left eye. The intraocular pressure was normal in both eyes. Bilateral slit lamp examination showed subtle, widespread corneal opacities, anterior lenticonus, and mild cataract. Bilateral dilated fundus evaluation revealed multiple yellowish lesions of differing sizes located along the superior vascular arcades, nasally to the optic discs, and in the temporal macular regions; the largest lesion constituted two-thirds of an optic disc diameter (Fig. 1a, b). Neither dot-and-fleck retinopathy nor diabetic retinopathy was present. Fundus autofluorescence imaging exposed hyperautofluorescence corresponding to the yellow lesions (Fig. 1c, d). On spectral-domain optical coherence tomography (SD-OCT) of the lesions, there was subretinal deposition of hyperreflective material at the level of the retinal pigment epithelium. Fluorescein angiography did not indicate associated leakage. Full-field electroretinography demonstrated slightly reduced photopic amplitudes. The electro-oculogram (EOG) was normal. Macular SD-OCT displayed multilayered retinoschisis located in the superotemporal macula of the left eye (Fig. 1e). Furthermore, central macular thinning and an irregular foveal contour were evident in both eyes (Fig. 2).

Due to anterior lenticonus, the patient underwent bilateral refractive lens exchange. Three months postoperatively, BCVA had improved to $6 / 9.5$ in the right eye $(6 / 7.5$ with pinhole) and 6/9.5 in the left eye (6/6 with pinhole). 


\section{Discussion}

The patient presented with symptomatic anterior lenticonus, a pathognomonic manifestation of AS. There were also structural changes on macular OCT in the form of retinoschisis, irregular foveal contour, and central retinal thinning, which have rarely been documented in AS $[3,4]$. Finally, multimodality retinal imaging confirmed multiple vitelliform lesions, a finding that has not been reported previously in AS.

The most prevalent retinal feature of AS is dot-and-fleck retinopathy [5]. Moreover, several less common retinal manifestations of AS have been described: bull's eye maculopathy [ 6 , $7]$, retinoschisis $[3,8,9]$, temporal retinal thinning $[5,10]$, "lozenge" or dull macular reflex $[4$, 6], macular holes [3, 6], peripapillary angioid streaks [9], retinal pigmentary maculopathy [6], and foveal "stair case pattern" [4]. There are also a few case reports of single vitelliform lesions in AS [3, 7]. With exception of macular holes and bull's eye maculopathy, these abnormalities have not been demonstrated to significantly impair vision [1]. Retinal manifestations of AS may therefore go unnoticed.

Although unifocal vitelliform lesions can manifest in AS, they represent a relatively prevalent, nonspecific finding that appears in several disorders, including Best disease (BD), adultonset vitelliform macular dystrophy, and various acquired conditions, including age-related macular degeneration. Multifocal vitelliform lesions, on the other hand, are much less common but do occasionally occur in atypical cases of BD [11], acute exudative polymorphous vitelliform maculopathy (AEPVM), and paraneoplastic vitelliform maculopathy (PVM). However, the patient had no family history of BD, and there was lack of central macular lesions or EOG findings arguing for BD. With regard to AEPVM, the medical history was negative for acute visual deterioration or prodrome symptoms of a viral infection. Finally, concurrent malignant disease pointing to PVM was not present. Their unusual occurrence and absence of plausible alternatives suggest that the multiple vitelliform lesions represent an ocular manifestation of AS.

The exact pathophysiology of the multiple vitelliform lesions found in the present patient is unknown. Yet, his underlying genetic condition raises the question about a link between structural changes in type IV collagen and retinal accumulation of vitelliform material. This particular collagen subtype contributes to the strength and integrity of the retinal internal limiting membrane (ILM) and Bruch's membrane (BM). A healthy ILM prevents vitreous traction and entrance of toxins to the retina, while BM under normal conditions regulates the passage of nutrients and metabolites between the RPE and underlying choriocapillaris [1]. The AS mutations lead to the deficiency of the physiological collagen IV $\alpha 3 \alpha 4 \alpha 5$ network. Instead, an immature $\alpha 1 \alpha 1 \alpha 2$ network causes formation of abnormal basement membranes [1, 5]. It is not fully understood how this affects the retina, but collagen IV $\alpha 1 \alpha 1 \alpha 2$ is found to have fewer cross-links, more proteolytic cleavage sites, and shows increased susceptibility to biomechanical strain [1]. These structural changes to collagen may explain why affected basement membranes appear as thinned or lamellated [5, 6]. Furthermore, increased permeability of an abnormal BM may result in accumulation of metabolic by-products and formation of vitelliform lesions $[3,5]$.

\section{Conclusion}

The present case report suggests that AS can manifest with multiple vitelliform lesions and adds to the evidence that ophthalmic features of AS are heterogeneous. The underlying 
genetic condition indicates that the pathophysiology of the vitelliform lesions is presence of a dysfunctional BM owing to abnormal type IV collagen.

\section{Statement of Ethics}

The patient has given written informed consent for publication of this case report.

\section{Disclosure Statement}

None of the authors have any conflicting interest to disclose.

\section{Funding Sources}

The authors did not receive any financial support.

\section{Author Contributions}

Dr. Kathrine O. Eriksen: patient assessment, data collection and analysis, literature search, manuscript drafting, and revision. Dr. Øystein Kalsnes Jørstad: data analysis and interpretation, design of the study, manuscript editing and revision, as well as final approval. Both authors contributed equally to the manuscript and are accountable for all aspects of the work.

\section{References}

1 Savige J, Sheth S, Leys A, Nicholson A, Mack HG, Colville D. Ocular features in Alport syndrome: pathogenesis and clinical significance. Clin J Am Soc Nephrol. 2015 Apr;10(4):703-9.

2 Kashtan CE. Alport syndrome. An inherited disorder of renal, ocular, and cochlear basement membranes. Medicine (Baltimore). 1999 Sep;78(5):338-60.

3 Thomas AS, Baynham JT, Flaxel CJ. Macular holes, vitelliform lesions, and midperipheral retinoschisis in Alport syndrome. Retin Cases Brief Rep. 2016;10(2):109-11.

4 Stanojcic N, Raja MS, Burton BJ. Choroidal thinning and "stair-case" foveal sign in a patient with Alport syndrome. Retin Cases Brief Rep. 2014;8(1):52-5.

5 Savige J, Liu J, DeBuc DC, Handa JT, Hageman GS, Wang YY, et al. Retinal basement membrane abnormalities and the retinopathy of Alport syndrome. Invest Ophthalmol Vis Sci. 2010 Mar;51(3):1621-7.

6 Savige J, Wang Y, Crawford A, Smith J, Symons A, Mack H, et al. Bull's eye and pigment maculopathy are further retinal manifestations of an abnormal Bruch's membrane in Alport syndrome. Ophthalmic Genet. 2017 May-Jun;38(3):238-44.

7 Fawzi AA, Lee NG, Eliott D, Song J, Stewart JM. Retinal findings in patients with Alport Syndrome: expanding the clinical spectrum. Br J Ophthalmol. 2009 Dec;93(12):1606-11.

8 Moisseiev E, Barak A. Bilateral macular retinoschisis in a patient with x-linked alport syndrome. Retin Cases Brief Rep. 2012;6(1):4-6.

9 Doménech-Aracil N, Hernández-Bel L, Castro-Navarro V, Barrios-Poleo C, Hernández Garfella M, CerveraTaulet E. New peripapillary findings in Alport syndrome: A case report. Arch Soc Esp Oftalmol. 2015 Oct;90(10):497-8.

10 Ahmed F, Kamae KK, Jones DJ, Deangelis MM, Hageman GS, Gregory MC, et al. Temporal macular thinning associated with X-linked Alport syndrome. JAMA Ophthalmol. 2013 Jun;131(6):777-82.

11 Boon CJ, Klevering BJ, den Hollander AI, Zonneveld MN, Theelen T, Cremers FP, et al. Clinical and genetic heterogeneity in multifocal vitelliform dystrophy. Arch Ophthalmol. 2007 Aug;125(8):1100-6. 


\section{Case Reports in Ophthalmology}
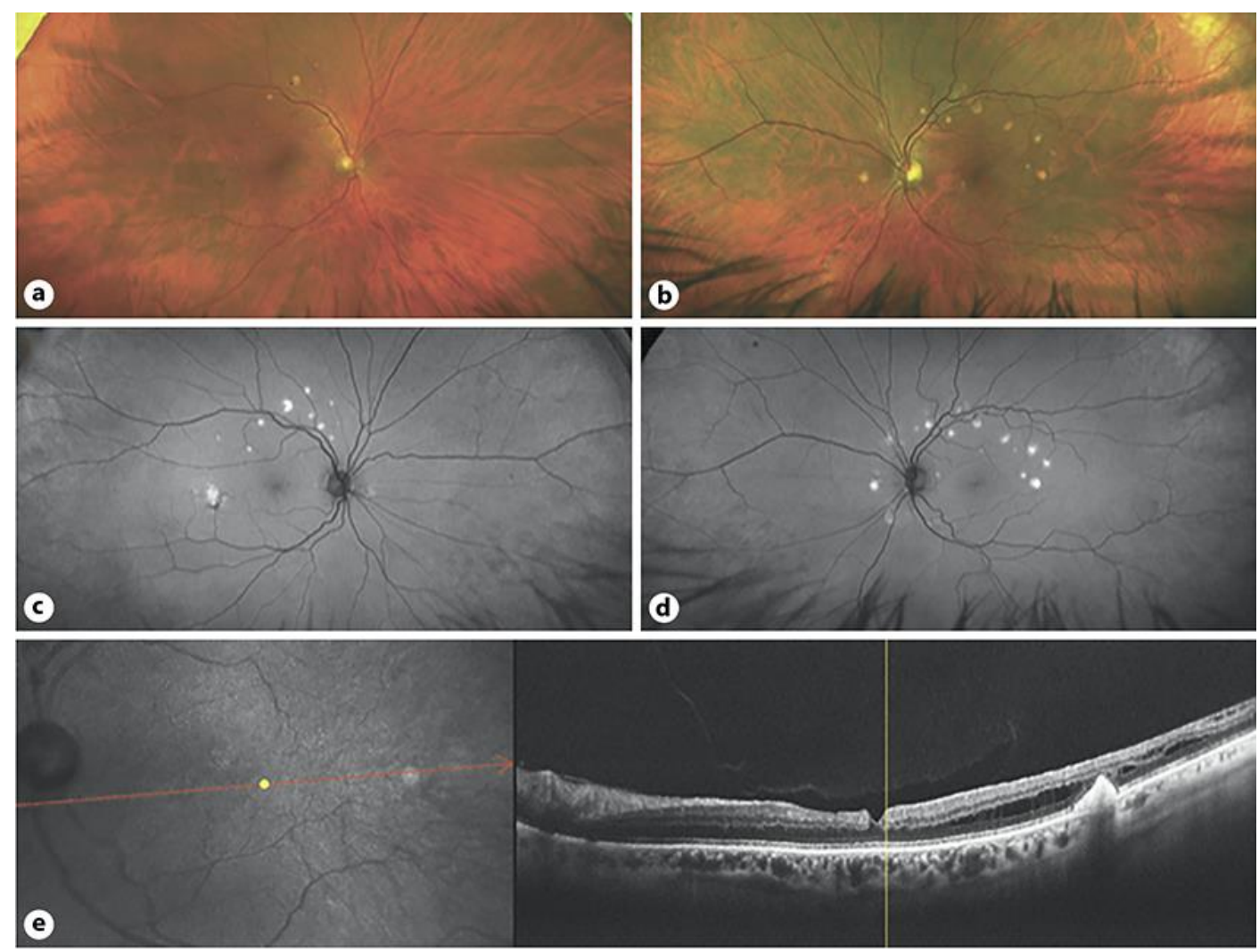

Fig. 1. Multimodal imaging of multiple vitelliform lesions in a patient with Alport syndrome. Scanning laser ophthalmoscopy of the right (a) and left (b) eye shows multiple yellowish lesions along the superior vascular arcades, in the temporal maculae, and nasally to the optic discs. Autofluorescence images of the right (c) and left (d) eye display hyperautofluorescence corresponding to the lesions. e Spectral-domain optical coherence tomography of the left macula reveals temporal disruption of the retinal pigment epithelium and hyperreflective subretinal material surrounded by a localized retinoschisis. There is also an irregular foveal contour. 


\section{Case Reports in Ophthalmology}

\begin{tabular}{l|l}
\hline Case Rep Ophthalmol 2020;11:79-84 \\
\hline DOI: 10.1159/000505948 & $\begin{array}{l}\text { @ 2020 The Author(s). Published by S. Karger AG, Basel } \\
\text { www.karger.com/cop }\end{array}$ \\
\hline
\end{tabular}

Eriksen and Jørstad: Multiple Vitelliform Lesions as a Retinal Manifestation of Alport Syndrome
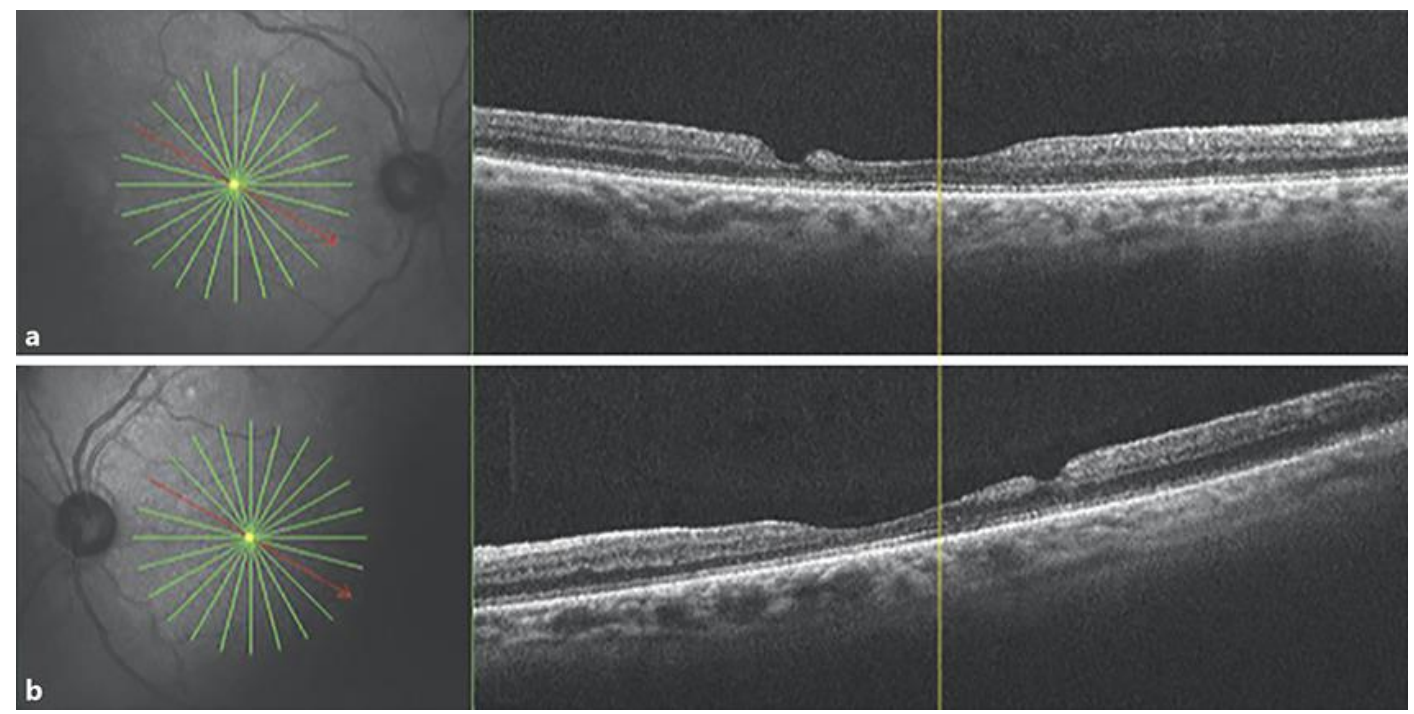

Fig. 2. Macular spectral-domain optical coherence tomography in a patient with Alport syndrome. In both the right (a) and the left (b) eye, there is central macular thinning and an irregular foveal contour. 